\title{
Una propuesta para la implementación de instrumentos técnicos y conceptuales en la construcción del objeto matemático: Área de figuras planas ${ }^{1}$
}

\author{
A proposal for the implementation of technical and conceptual tools in \\ the construction of the mathematical object: area of plane figures \\ A proposta para a implementação de ferramentas técnicas e conceituais \\ para a construção do objeto matemático: área de figuras planas
}

Recibido: mayo de 2013

Aceptado: agosto de 2013

Julián Humberto Santos ${ }^{2}$

\section{Resumen}

La siguiente propuesta tiene como fin incentivar el trabajo docente en el campo de la educación matemática, frente a la implementación en el aula de instrumentos técnicos y conceptuales que faciliten la construcción de objetos matemáticos. Estos procesos de implementación, son un recurso casi indispensable para captar la atención de estudiantes, que han incorporado a su desarrollo social y cultural de forma total el entorno tecnológico. Cuando un docente es capaz de incorporar en el aula instrumentos conceptuales de la mano con instrumentos técnicos, logra generar en su estudiante el gozo de ser constructor de su propio conocimiento.

Palabras clave: Gestión del aula; Instrumentos; Recursos didácticos; Aula; Recursos didácticos; Recursos informáticos; Software; Matemáticas escolares; Geometría.

\begin{abstract}
The following proposal is intended to stimulate the teaching work in the field of mathematics education, compared to classroom implementation of technical and conceptual tools that facilitate the construction of mathematical objects. These implementation processes are almost indispensable resource to capture the attention of students, who have incorporated social and cultural development in whole technological environment. When a teacher is able to incorporate in the classroom hand conceptual tools with technical tools, able to generate in your student the joy of being a builder of their own knowledge.
\end{abstract}

Keywords: Classroom Management, Instruments Educational resources, Classroom, Teaching Resources, Computer Resources, Software, Mathematics school; Geometry.

\section{Resumo}

A seguinte proposta visa estimular o trabalho docente no campo da educação matemática, em comparação com a implementação em sala de aula

1 Artículo de Investigación.

2 Agustiniano TagasteSchool. Contacto: guly16@hotmail.com. 
de ferramentas técnicas e conceituais que facilitam a construção de objetos matemáticos. Estes processos de implementação são recursos quase indispensável para captar a atenção dos alunos, que têm incorporado o desenvolvimento social e cultural em todo ambiente tecnológico. Quando um professor é capaz de incorporar nas ferramentas conceituais mão em sala de aula com ferramentas técnicas, capazes de gerar em seu aluno a alegria de ser um construtor de seu próprio conhecimento.

Palavras-chave: Gestão de Sala de Aula, Recursos educativos Instruments, Sala de Aula, Recursos de Ensino, Recursos Informática, Software, escola Matemática, Geometria.

\section{Contextualización}

La utilización de instrumentos técnicos en el aula, cobra vital importancia a la hora de construir conceptos complejos, esto, por el dinamismo que presenta el instrumento al momento de palpar la teorización de las nociones y por sus facilidades al momento de manipular el significado de un objeto matemático.

En la actualidad la educación matemática se plantea un desafío frente a la necesidad de llevar de la mano al salón de clase, los innumerables instrumentos técnicos y conceptuales que constituyen el día a día de la labor docente, generando al mismo tiempo una construcción del conocimiento en el aula. Como lo menciona Llinares (2008), las personas pensamos y actuamos ayudadas por instrumentos, por eso se fundamenta el uso del instrumento con el fin de planificar actividades que constituyan la práctica de enseñar matemáticas. Bajo esta afirmación nace la necesidad de generar procesos de enseñanza-aprendizaje que apunten a la implementación de instrumentos en el aula.

Ésta intervención se implementó con estudiantes del grado 8 del colegio Agustiniano Tagaste, ubicado en la ciudad de Bogotá, con el fin de construir el objeto matemático: área y perímetro de figuras planas, a partir de la utilización de un instrumento técnico (computadores portátiles serie Educa, Norma), haciendo uso de las plataformas de red $\mathrm{y}$ otras herramientas del instrumento, dando respuesta a la pregunta $i$ Influye positivamente en la gestión del conocimiento conceptual del docente, la implementación de instrumentos técnicos y conceptuales en la construcción de un objeto matemático?

\section{Referentes teórico prácticos}

Autores como Linares (2008) aseguran que las perspectivas socioculturales del aprendizaje ampliaron el significado de instrumento como un objeto físico para incluir también conceptos, formas de razonar y formas de generar un discurso, además clasifica el instrumento en dos: instrumentos técnicos, como por ejemplo materiales y recursos didácticos, geo planos o software e instrumentos conceptuales, constituidos por los conocimientos conceptuales que fundamentan la práctica de enseñar matemáticas.

$\mathrm{Al}$ hacer uso de estos instrumentos, es posible fortalecer los procesos de enseñanza - aprendizaje en el campo de la construcción geométrica, como lo indica Guzmán (1993), la matemática requiere de lo intuitivo y visual, generando para ello dos tipos de habilidades: la primera, la visualización y representación de figuras: leer, interpretar y comprender, construcciones que se encuentran en el medio y que le permitirán contextualizar su conocimiento, la segunda, un procesamiento mental de las imágenes, que le dará la de manipular y transformar los conceptos relacionados con dichas imágenes en un 
conocimiento más elaborado, lo cual se desarrolló dentro de la experiencia con la construcción del solido geométrico y la modelación de su módulo.

La constitución del conocimiento conceptual refiere al hecho de realizar un análisis mas profundo de las situaciones que encierran los procesos de enseñanza-aprendizaje, generando conceptos y construcciones conceptuales que permitan la reflexión frente al proceso. Reconocer si existe una incidencia positiva frente a este proceso de constitución del conocimiento conceptual desde un instrumento técnico fue la fundamentación práctica de este estudio.

Es necesario entonces considerar que la matemática educativa al igual que diversas áreas del conocimiento, han incorporado a su desarrollo el tratamiento de nuevas tecnologías, estas tecnologías constantemente están generando nuevas redes de conocimiento, que proporcionan nuevas herramientas para el diseño de materiales didácticos favoreciendo los espacios de interacción entre sujetos.

\section{Descripción de la experiencia}

El objeto matemático escogido fue: construcción del área y perímetro de figuras planas a partir de la construcción de un Icosaedro usando papiroflexia modular. Los estudiantes debían en primera instancia construir el Icosaedro, teniendo como base los pasos y modelos planteados por Velasquez (2009) quien presenta una propuesta metodológica para promover la construcción en los estudiantes, de algunas nociones geométricas con relación a la geometría plana y tridimensional. Una vez desarrollada la construcción los estudiantes debían descomponer el modulo utilizado en el armado de la figura, esto, para evidenciar las figuras geométricas y establecer sus áreas y perímetros.

Es allí donde el instrumento técnico cobra vital importancia en la construcción del objeto matemático. Los estudiantes por iniciativa propia y tras una previa revisión de algunos software libres para el desarrollo matemático, deciden modelar el modulo utilizando el instrumento, generando así la implementación y concreción tanto del instrumento conceptual como del instrumento técnico en el aula de clase.

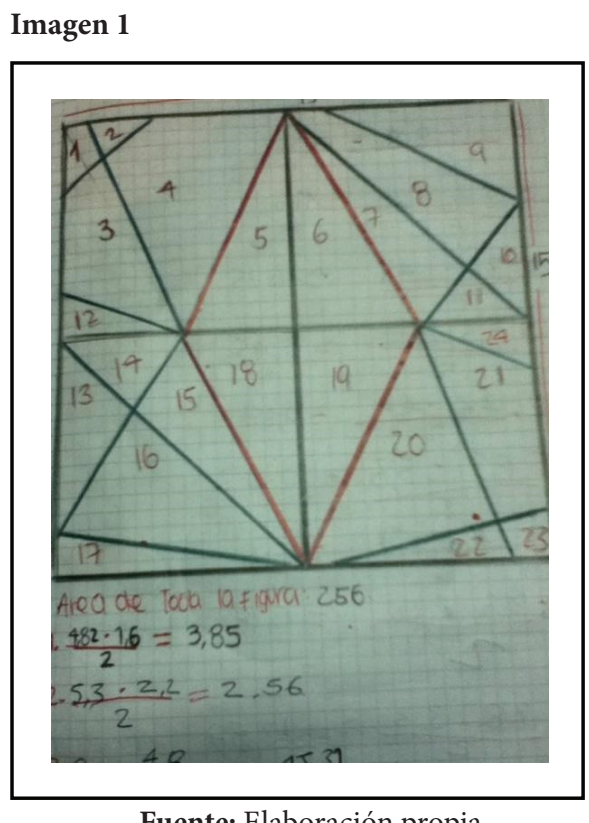

Los estudiantes lograron establecer y adaptar un instrumento que permitiera modelar su representación gráfica, logrando además generar un cambio de registro desde lo grafico a lo meramente verbal con el fin de compartir su sorpresivo avance en el campo de las representación a partir de un software.

\section{Logros y dificultades}

El aporte generado a la construcción del objeto matemático, por la implementación a la propuesta de un instrumento técnico por parte del estudiante, permitió en ellos los siguientes logros:

- Construcción del objeto matemático: áreas de figuras planas a partir de la elaboración de un Icosaedro modular.

- Inmersión en las nuevas tecnologías educativas, a partir del uso y manejo de software y plataformas en el aula de clase. 
- El Gozo de construir por si mismo y a partir de su autorreflexión y autoconocimiento el objeto matemático abordado.

- La posibilidad de verificar los procesos aritméticos construidos en el papel, mediante el uso de instrumentos.

- La generación de posibles comunidades de práctica, conformadas por estudiantes, que tendrían como fin mejorar los procesos educativos llevados a cabo en el aula.

- Inquietar el espíritu en ocasiones dormido, de estudiantes acostumbrados a la linealidad de la matemática, que vieron en la propuesta una oportunidad para hacer de las matemáticas su estilo de vida.

\section{Imagen 2}

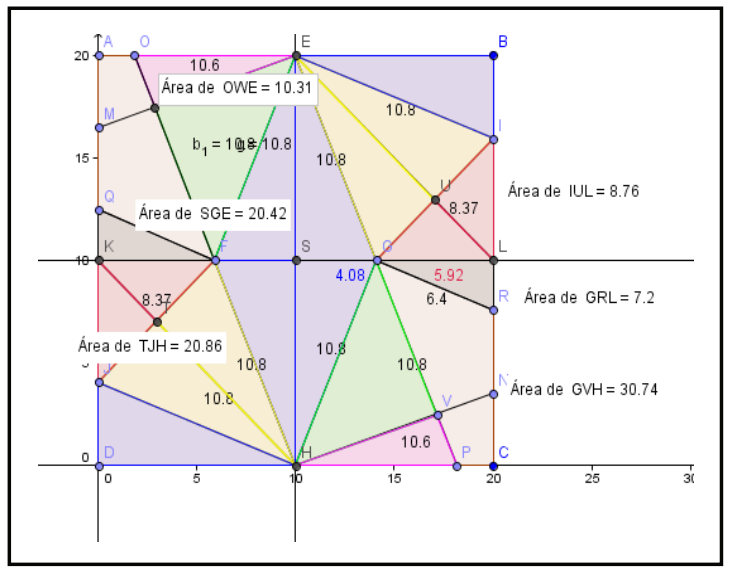

Fuente: Elaboración propia

Aunque los logros fueron bastantes y las experiencias enriquecedoras para la observación de la práctica docente, como todo proceso investigativo se presentaron algunas dificultades que en un futuro mejoraran las propuestas implementadas:

- El nivel de los estudiantes frente al manejo de instrumentos técnicos en el aula no es equitativo, algunos desconocen por completo software y herramientas que podrían mejorar su proceso educativo.

- Algunos estudiantes se han acostumbrado a observar las matemáticas como un proceso lineal, cerrándose a la oportunidad de construir conocimiento a partir del trabajo con instrumentos técnicos y conceptuales.

\section{Reflexión final}

La implementación de instrumentos técnicos en la construcción del objeto matemático: áreas de figuras planas, permitió la participación activa de los estudiantes que se vieron atraídos, persuadidos e interesados por inmiscuirse en la generación de un aprendizaje significativo, que plasma su interés por las nuevas tecnologías.

Frente a la constitución del conocimiento conceptual docente, la inmersión en el aula de instrumentos técnicos permite reflexionar sobre la verdadera

Imagen 3



Fuente: Elaboración propia 
construcción de conocimiento en el aula. El docente debe estar a la vanguardia de las nuevas tecnologías en el aula y asumir estas como instrumento que permitan la generación de aprendizaje significativo, estar aun en una postura reacia a esta necesidad, significa estar en contra de un cambio necesario y efectivo. Los instrumentos conceptuales pueden estar en continua relación con el instrumento técnico y permitir un empalme que optimice resultados.

La utilización de los instrumentos genero en los estudiantes una zona de confort, donde sabían lo que hacían, por que estaban dentro del gusto de su cotidianidad: la tecnología, sus argumentos fueron mas solidos, sus posturas mas argumentadas y su sistema de representación verbal fue mas elaborado que en otras situaciones.

La gran cantidad de programas matemáticos existentes en la actualidad, para el desarrollo del algebra, el cálculo, la trigonometría, la aritmética y la geometría son un paso importante en la búsqueda de la abolición del prejuicio escolar de observar a las matemáticas como castigo educativo, sin embargo su desconocimiento hace que maestros y estudiantes no se unan a su uso y su discusión, es por ello que considerar cada vez con mayor profundidad estos elementos hará que nos relacionemos más con sus beneficios y nos hagamos participes de sus ventajas.

\section{Referencias}

Cabello, Lili G (2006). La Enseñanza De La Geometría Aplicando Los Modelos de Recreación y Reflexión a Través de la Funcionalidad de Materiales Educativos: $V$ festival internacional de matemáticas.

Fajardo, N. Velasquez, L. (2009). Propuesta metodológica para la enseñanza de la geometría a través de la papiroflexia. X encuentro de matemática educativa, ASOCOLME. Pasto. Colombia.

Llinares, S. (2008) Aprendizaje del estudiante para profesor de matemáticas y el papel de los nuevos instrumentos de comunicación. Conferencia Universidad Pedagógica Nacional. Bogotá. Colombia.

Llinares, S. (s.f) Intentando comprender la práctica del profesor de matemáticas. Departamento de didáctica de las matemáticas. Universidad de Sevilla 